\title{
Correction to: Safety and effectiveness of vedolizumab in paediatric patients with inflammatory bowel disease: an observational multicenter Spanish study
}

\author{
Ruth Garcia-Romero ${ }^{1}$ (D) - José Miguel Martinez de Zabarte Fernandez ${ }^{2}$. \\ Gemma Pujol-Muncunill $^{3}$. Ester Donat-Aliaga ${ }^{4}$ • Oscar Segarra-Cantón ${ }^{5}$. \\ Iñaki Irastorza-Terradillos ${ }^{6}$ - Enrique Medina-Benitez ${ }^{7}$ - Carlos José Ruiz-Hernández ${ }^{8}$. \\ Marta Carrillo-Palau ${ }^{9}$ • Ignacio Ros-Arnal ${ }^{1}$ • Alejandro Rodriguez-Martínez ${ }^{10}$. \\ Laura Escartin-Madurga $^{11}$ - Carolina Gutiérrez-Junquera ${ }^{12}$ - Saioa Vicente-Santamaría ${ }^{13}$. \\ Marta Velasco Rodriguez-Belvis ${ }^{14}$. Sonia Fernández-Fernández ${ }^{15}$. \\ José Ramón Alberto-Alonso ${ }^{16}$. Montserrat Montraveta ${ }^{17} \cdot$ Ricardo Torres-Peral $^{18}$. \\ María Navalon-Rubio ${ }^{19}$. Víctor Manuel Navas-López ${ }^{20} \cdot$ Javier Martin de Carpi $^{3}$
}

Published online: 23 May 2021

C Springer-Verlag GmbH Germany, part of Springer Nature 2021

Correction to: European Journal of Paediatrics https://doi.org/10.1007/s00431-021-04063-6

In the original published version of this article under the last part of the Abstract section the author made changes on their presented data the corrected data are as follows [Bold text used to highlight problem area]:

\section{Abstract}

In the sentence, "At week 14, $69 \%$ of the patients responded to the treatment $(57.1 \%$ of those with $\mathbf{U C}$ and $75 \%$ of those with $\mathrm{UC} ; \mathrm{p}=0.238)^{\prime \prime}$
The correct version should have been: "At week 14, 69\% of the patients responded to the treatment $(57.1 \%$ of those with CD and $75 \%$ of those with UC; $\mathrm{p}=0.238) "$.

The original article has been corrected.

The online version of the original article can be found at https://doi.org/ 10.1007/s00431-021-04063-6

Ruth Garcia-Romero

ruthgarciaromero@yahoo.es

Extended author information available on the last page of the article 


\section{Affiliations}

\section{Ruth Garcia-Romero ${ }^{1}$ (D) - José Miguel Martinez de Zabarte Fernandez ${ }^{2} \cdot$ Gemma Pujol-Muncunill $^{3}$. \\ Ester Donat-Aliaga ${ }^{4}$ • Oscar Segarra-Cantón ${ }^{5}$ • Iñaki Irastorza-Terradillos ${ }^{6}$ • Enrique Medina-Benitez ${ }^{7}$. \\ Carlos José Ruiz-Hernández ${ }^{8}$ • Marta Carrillo-Palau ${ }^{9} \cdot$ Ignacio Ros-Arnal $^{1}$ • Alejandro Rodriguez-Martínez ${ }^{10}$. Laura Escartin-Madurga ${ }^{11}$ • Carolina Gutiérrez-Junquera ${ }^{12}$ • Saioa Vicente-Santamaría ${ }^{13}$. \\ Marta Velasco Rodriguez-Belvis ${ }^{14}$. Sonia Fernández-Fernández ${ }^{15}$ • José Ramón Alberto-Alonso ${ }^{16}$. Montserrat Montraveta ${ }^{17} \cdot$ Ricardo Torres-Peral $^{18} \cdot$ María Navalon-Rubio $^{19}$ • Víctor Manuel Navas-López ${ }^{20}$. Javier Martin de Carpi ${ }^{3}$}

1 Paediatric Gastroenterology, Hepatology and Nutrition, Paediatric University Hospital Miguel Servet, Zaragoza, Spain

2 Paediatric Gastroenterology and Nutrition, Hospital Obispo Polanco, Teruel, Spain

3 Paediatric Gastroenterology, Hepatology and Nutrition, Hospital Sant Joan de Déu, Barcelona, Spain

4 Paediatric Gastroenterology, Hepatology and Nutrition, Polytechnic University Hospital La Fe, Valencia, Spain

5 Paediatric Gastroenterology, Hepatology and Nutrition, MotherChild University Hospital, Vall Hebrón, Barcelona, Spain

6 Paediatric Gastroenterology, Hepatology and Nutrition, University Hospital of Cruces, Barakaldo, Spain

7 Paediatric Gastroenterology, Hepatology and Nutrition Unit, University Hospital 12 de Octubre, Madrid, Spain

8 Paediatric Gastroenterology and Nutrition, Department of Paediatrics, Hospital Parc Taulí, Sabadell, Spain

9 Department of Gastroenterology, University Hospital of the Canary Islands, La Laguna, Tenerife, Spain

10 Paediatric Gastroenterology, Hepatology and Nutrition, University Hospital Virgen del Rocío, Sevilla, Spain
11 Paediatric Gastroenterology, Hepatology and Nutrition, University Clinic Hospital Lozano Blesa, Zaragoza, Spain

12 Paediatric Gastroenterology, Hepatology and Nutrition, Hospital Universitario Puerta de Hierro-Majadahonda, Madrid, Spain

13 Paediatric Gastroenterology, Hepatology and Nutrition, University Hospital Ramon y Cajal, Madrid, Spain

14 Paediatric Gastroenterology, Hepatology and Nutrition, Hospital Niño Jesús, Madrid, Spain

15 Paediatric Gastroenterology, Hepatology and Nutrition, University Hospital Severo Ochoa, Leganés, Madrid, Spain

16 Paediatric Gastroenterology, Hepatology and Nutrition, University Hospital Ntra. Sra. de Candelaria, Tenerife, Spain

17 Paediatric Gastroenterology, Hepatology and Nutrition, Hospital Germans Trias i Pujol, Badalona, Spain

18 Paediatric Gastroenterology and Nutrition Unit, Department of Paediatrics, University Hospital Complex, Salamanca, Spain

19 Paediatric Gastroenterology, Hepatology and Nutrition, Hospital Virgen de la Arrixaca, Murcia, Spain

20 Paediatric Gastroenterology and Nutrition Section, Regional University Hospital of Malaga, Málaga, Spain 